\title{
EVALUACIÓN DE LA RESISTENCIA A LA MUSTIA HILACHOSA (Thanatephorus cucumeris (Frank), Donk). EN LÍNEAS Y VARIEDADES DE FRIJOL. CAISAN. PANAMÁ. 19971
}

\author{
Emigdio Rodríguez ${ }^{2}$, Francisco González ${ }^{3}$
}

\begin{abstract}
RESUMEN
Evaluación de la resistencia a la mustia hilachosa (Thanatephorus cucumeris (Frank), Donk). en líneas y variedades de frijol. Caisán. Panamá, 1997. Con el propósito de identificar genotipos de frijol común, con un nivel mayor de resistencia a la mustia hilachosa se estableció la presente investigación, en Caisán, Panamá. Se evaluaron 4335 líneas y variedades de frijol común provenientes de siete ensayos. Se utilizaron los diseños experimentales de bloques completos al azar y látice simple, según el ensayo, con una, dos o tres repeticiones, lo que dependió de la cantidad de semilla disponible. Hubo 62 materiales con reacción inferior a la mustia hilachosa en relación con el mostrado por el testigo resistente Talamanca (grado 5,0). El testigo susceptible Barriles mostró grado de reacción 8,0 a este patógeno. Se observó una aparente reacción de hipersensibilidad a la mustia hilachosa en varias líneas.
\end{abstract}

\begin{abstract}
Resistance sources to the web blight (Thanatephorus cucumeris) (Frank), Donk). Caisán. Panama, 1997. This study was carried out with the purpose of identifying genotypes of common bean, with a greater level of resistance to the web blight, in Caisán, Panama. 4335 lines and varieties of common bean were evaluated from seven experiments. A completely randomized design and a simple lattice design were utilized according to the experiments. Experiment repetitions varied from one, two or three depending on the amount of seed available. 62 materials showed an inferior reaction to the web blight in relation to that of the resistant Talamanca control (5 grade). The Barriles susceptible control showed grade reaction of 8 . In several lines it was observed an apparent reaction of hyper-sensitivity to the web blight.
\end{abstract}

\section{INTRODUCCIÓN}

En Panamá la producción de frijol se da principalmente en el occidente de la Provincia de Chiriquí, donde se cultiva alrededor del $95 \%$ de la producción nacional (Rodríguez, Gracia y González, 1995). En esas condiciones las principales limitantes del sistema productivo son las malezas, la falta de variedades mejoradas, y enfermedades como la mustia hilachosa y la mancha angular.

La mustia hilachosa del frijol común (Phaseolus vulgaris $\mathrm{L}$ ) es causada por el hongo Rhizoctonia solani Kuhn, el estado esclerocial o asexual del basidiomiceto Thanatephorus cucumeris (Frank), Donk (Galvez, Mora y Pastor, 1994). Está ampliamente distribuido en todo el mundo. Tanto su estado esclerocial como basidial pueden iniciar la enfermedad, aunque los síntomas son diferentes; en la mayoría de los lugares de América
Latina donde la enfermedad se presenta, el estado eclerocial es importante para la iniciación y la diseminación de la misma (Galindo, 1982).

La mustia hilachosa es un problema muy importante para la producción de frijol en las tierras tropicales bajas y húmedas de América Latina y El Caribe, donde predominan las temperaturas moderadas a cálidas y una precipitación abundante. También puede causar daños severos en zonas de altitud media (1200-1600 msnm), en particular cuando el clima es lluvioso y la humedad alta. (Galvez, Mora y Pastor, 1994). Los primeros síntomas aparecen en las hojas y se presentan como pequeñas lesiones acuosas circulares de 1-3- mm de diámetro y de coloración más clara que la hoja (Pastor, 1985).

La resistencia genética contra Rhizoctonia solani es considerada como la mejor alternativa de control, sin embargo los esfuerzos invertidos a la fecha no han

\footnotetext{
1 Presentado en la XLIV Reunión Anual del PCCMA, Nicaragua, 1998. Financiado parcialmente por PROFRIJOL.

2 Fitomejoramiento. Gerente del Proyecto de Frijol en Panamá. IDIAP.

3 IDIAP. Panamá.
} 
dado los frutos esperados (Beebe, 1986), debido fundamentalmente a que estos esfuerzos no han sido consistentes en el tiempo y ha estado orientado por investigadores que tampoco han sido consistentes en sus trabajos.

Los cultivares de frijol difieren en su reacción al patógeno de la mustia hilachosa en condiciones de campo; los cultivares susceptibles exudan productos químicos que estimulan la formación de cojines de infección, mientras que los cultivares resistentes o tolerantes no exudan estos productos (Flentje, Dodman y Kerr, 1963).

Con anterioridad se han identificado algunas fuentes de resistencia a esta enfermedad evaluando accesiones al azar y que por simple coincidencia fueron encontradas. Algunas de estas fuentes son las líneas AFR-251 y PVA - 800 de donde se originó la línea I-44 con un nivel de tolerancia mayor que las variedades comerciales. Esto fue producto de cruzas simples pero existe la sospecha de que son varios genes los que conceden la resistencia a este patógeno y si se desea incrementar los niveles encontrados a la fecha, no podrá ser de otra manera sino a través de la piramidación de genes de resistencia (Rodríguez y González, 1998).

El programa Regional de Frijol (PROFRIJOL), realiza esfuerzos con miras a encontrar resistencia genética a esta enfermedad y desarrolla trabajos en Panamá, República Dominicana y Puerto Rico. Las actividades emprendidas son: la realización de cruzamientos, evaluación de poblaciones segregantes en generaciones tempranas y la evaluación de viveros específicos. En los últimos años se ha podido constatar la existencia de variabilidad genética, la que se esta utilizando favorablemente en la lucha contra esta enfermedad. (PROFRIJOL, 1997).

La evaluación sistemática de los genotipos de frijol no se había realizado con anterioridad, hasta este año cuando el Proyecto de Frijol de Panamá en conjunto con el PROFRIJOL deciden evaluar a colección más representativa del frijol del mundo existente en la región.

Los objetivos de la presente investigación fueron: Identificar genotipos con un nivel mayor de resistencia a la mustia hilachosa que la mostrada por las variedades utilizadas como testigos tolerantes, e identificar progenitores adecuados que permitan aumentar el nivel de resistencia a la mustia hilachosa.

\section{MATERIALES Y MÉTODOS}

Los ensayos se establecieron en Caisán, Distrito de Renacimiento, Provincia de Chiriquí, República de Panamá. Caisán tiene una altura promedio de $800 \mathrm{msnm}$ aproximadamente con una temperatura y humedad relativa de $22^{\circ} \mathrm{C}$ y $80 \%$ respectivamente.

Se evaluaron un total de 4335 líneas de frijol común por su reacción a la mustia hilachosa las cuales provenían de los viveros: Colección núcleo del CIAT (CORE), Vivero internacional de frijol (IBN), Vivero fuentes de Resistencia (VIFURE), Vivero de adaptación de grano rojo y grano negro (VIDAC-rojo y VIDAC-negro) y Vivero internacional Caribeño (VICARIBE), materiales caribeños y líneas nacionales (cruza simple, cruzas triples). Cada experimento contó con un diseño experimental apropiado a cada ensayo lo que dependió del número de entradas y de la cantidad de semilla disponible, en general los diseños utilizados fueron látices simples y bloques completos al azar, con una, dos o tres repeticiones lo que dependió de la cantidad de semillas disponibles.

La preparación del suelo se realizó con el método de labranza convencional, que consistió en un pase de rastra con dos pases de arado rotativo y luego se aplicó el herbicida paraquat a una dosis de $0,40 \mathrm{~kg}$ i.a./ha a los 25 días después de la siembra en forma dirigida utilizando pantallas protectoras (Rodríguez et al., 1997).

Se fertilizó a la siembra con $113.4 \mathrm{~kg} / \mathrm{ha}$ de fertilizante $18-46-0$ y $181,44 \mathrm{~kg} / \mathrm{ha}$ de urea al $46 \%$ a los 35 días después de la siembra.

A la siembra se aplicó el insecticida Carbofuran $10 \% \mathrm{G}$ a una dosis de $20 \mathrm{~kg} / \mathrm{ha}$ para evitar daños a la plántula por ataque de insectos del suelo. No se hizo aplicación de fungicidas.

A los 25 días después de la siembra se hizo una aplicación foliar de inóculo de la mustia hilachosa para propiciar la enfermedad. Este inóculo fue preparado colectando hojas infectadas con el patógeno, se preparó un maserado de las mismas y posteriormente se aplicó a las plantas de frijol utilizando una bomba de motor para realizar esta aplicación.

Luego de la aplicación del inóculo se esperó hasta que la enfermedad apareciera en el testigo susceptible (Barriles) con una intensidad de un $70 \%$ del tejido foliar afectado por el patógeno para proceder a realizar la evaluación de todo el germoplasma . 
Se evaluó el grado de reacción de la líneas de frijol utilizando la escala internacional del CIAT, la cual se detalla a continuación:

\begin{tabular}{ccl}
\hline Grado & \% de sev & Reacción \\
\hline 1 & 0 & \\
2 & $1-5$ & Resistente \\
3 & $6-10$ & \\
4 & $11-20$ & Resistencia \\
5 & $21-30$ & \\
6 & $31-40$ & Intermedia \\
7 & $41-60$ & Susceptible \\
8 & $61-80$ & \\
9 & $81-100$ & \\
\hline
\end{tabular}

\section{RESULTADOS Y DISCUSIÓN}

\section{Evaluación de la Colección Núcleo "CORE” del CIAT.}

De la evaluación de 1305 líneas por su reacción al ataque del hongo que ocasiona la mustia hilachosa, sobresalieron ocho genotipos con un grado de tres, mientras que el testigo tolerante, variedad Talamanca, mostró un grado de reacción de cinco (Cuadro 1). Los genotipos en evaluación mantuvieron su reacción hasta el final de la fase reproductiva de la planta. Se observó en estas líneas una reacción aparente hipersensitiva ya que el hongo iniciaba su ataque sobre el tejido foliar de la planta, pero ésta formaba un halo de color marrón alrededor de la lesión que impedía que la enfermedad avanzara.

Es importante conocer la identificación correcta de estos genotipos y explorar estas fuentes que es donde se pudiese encontrar reacciones superiores a las encontradas en esta oportunidad. Estos materiales serán evaluados en laboratorio para confirmar su reacción. Estas líneas se evaluarán en varias localidades de diferentes países, para medir la reacción que se presentará en cada región.

Cuadro 1. Genotipos sobresalientes del "CORE" por su reacción a la Mustia Hilachosa.

\begin{tabular}{llc}
\hline Genotipo & Origen & Grado de reacción \\
\hline G-1844 & Costa Rica & 3 \\
G-2472 & México (Guerrero) & 3 \\
G-10075 & Holanda & 3 \\
G-13046 & México (Puebla) & 3 \\
G-14310 & Malawi & 3 \\
G-14380 & México (Puebla) & 3 \\
G-18454 & Nicaragua & 3 \\
BAT 93 & CIAT & 3 \\
BARRILES (T.S.) & Panamá & 8 \\
TALAMANCA (T.T.) & Costa Rica & 5 \\
\hline
\end{tabular}

El otro aspecto que es importante indicar es que el grado de reacción 3-0 a este patógeno nunca se había observado en los experimentos montados en Caisán.

\section{Evaluación del Vivero Internacional de Frijol "IBN".}

De la evaluación de 1106 líneas de frijol sobresalió la línea BRB-240 con reacción de 4,0 y las líneas Belmidak-RR-1, Belmidak - RR2, Belmidak - RR4, LRS 92-1, Fin -10 e I - 44 con reacción de 5,0. Los testigos en esta evaluación mostraron reacción de nueve para el testigo susceptible (Barriles) y siete para el testigo tolerante (Talamanca). Estos genotipos serán evaluados nuevamente en el siguiente ciclo agrícola para comprobar su reacción al hongo (Cuadro 2). De reconfirmarse la reacción de estos genotipos se podrán incluir como padres potenciales para futuros cruzamientos.

Cuadro 2. Líneas sobresalientes en el IBN por su reacción a la Mustia Hilachosa.

\begin{tabular}{lc}
\hline Genotipo & Reacción \\
\hline BRB 240 & 4 \\
Belmidak RR1 & 5 \\
Belmidak RR 2 & 5 \\
Belmidak RR4 & 5 \\
LRS 92-1 & 5 \\
FIN -10 & 5 \\
I-44 & 5 \\
Barriles (T.S.) & 9 \\
Talamanca (T.T.) & 7 \\
\hline
\end{tabular}

\section{Evaluación del Vivero de Fuentes de Resistencia "VIFURE"}

De las 143 líneas de frijol que conformaban este vivero por su reacción a la mustia hilachosa sobresalió la línea Belmidak RR9 con reacción de 4,0 a la mustia hilachosa la cual se mantuvo hasta el final del cultivo. La línea en cuestión debe de contener en su información genética genes que le permiten soportar mejor un fuerte ataque de la enfermedad. Esta línea mostró claramente la sintomatología de hipersensitividad que mostraron las líneas del "CORE". Es necesario mencionar que este vivero fue concebido para atender otras necesidades de la región sin embargo fue evaluado por su reacción a la mustia hilachosa y mostró un material con excelente adaptación a condiciones donde se presenta la mustia hilachosa. Este genotipo se volverá a sembrar en Caisán con el propósito de reconfirmar su reacción al patógeno que ocasiona esta enfermedad y de ser confirmada su reacción se podrá iniciar un programa de cruzamiento donde se incluirá esta línea como progenitor. 
Evaluación de Líneas F5 y F6 obtenidas por selección individual.

De la evaluación de 342 líneas F5 de cruzamientos triples y 801 líneas F6 provenientes de cruzamientos simples se seleccionaron cuatro líneas F5 con reacción de 4,0 y 5,0 de severidad de la mustia hilachosa, adicionalmente se seleccionaron cinco líneas, que aunque tenían reacción de seis, siete y ocho tenían plantas dentro del surco totalmente sanas y a las cuales se les practicó selección individual (Cuadro 4). De las líneas F6 se seleccionaron 31 familias con reacción de tres, cuatro y cinco y donde también se realizaron las selecciones individuales dentro de estas líneas ya que eran plantas totalmente sanas. Esta líneas formarán parte del VICARIBE para el siguiente ciclo donde se probarán por otros factores limitantes como son el mosaico dorado y bacteriosis común.

Evaluación del Vivero de Adaptación de Grano rojo y Grano negro (VIDAC- rojo y VIDAC - negro).

De las 99 líneas del grano rojo evaluadas solamente dos líneas mostraron reacción de resistencia intermedia dando un grado de reacción de cinco y seis para la evaluación de mustia hilachosa practicada en el ensayo; estas fueron las líneas SRC-1-1-18 y SRC-1- 4 - 4 en tanto que los testigos tolerantes y susceptibles (Talamanca y Barriles) mostraron reacción de nueve y siete en cada uno de los casos.

De las 89 líneas evaluadas en el VIDAC - negro únicamente una línea mostró reacción de 4.0 y fue el DOR-447, en tanto que DOR 620, DOR 617, ICTA-JU95-97, ICTA-JU-95-91, ICTA JU-95-56, ICTA-JU-9572, ICTA JU 95-47 y el DOR-647 mostraron una reacción de 6,0. Todas estas líneas mostraron resistencia intermedia a la mustia hilachosa. En los testigos tolerantes y susceptibles (Talamanca y Barriles) se encontró una reacción de susceptibilidad con evaluaciones de siete y nueve respectivamente.

Lo anterior es indicativo de que los genotipos negros han sido más evaluados que los rojos, sin embargo la fuente utilizada pareciera ser la misma y única, la que aporta muy poco a la resistencia a este patógeno (Cuadro 5).

Cuadro 3. Líneas sobresalientes por su reacción a la Mustia Hilachosa en la evaluación del VIFURE.

\begin{tabular}{lc}
\hline Genotipo & Grado de reacción \\
\hline Belmidak RR - & 4 \\
Barriles (T.S.) & 9 \\
Talamanca (T.T.) & 7 \\
\hline
\end{tabular}

Cuadro 4. Evaluación de líneas F5 y F6 por su reacción a la mustia hilachosa.

\begin{tabular}{ccc}
\hline \multicolumn{3}{c}{ Líneas F5 } \\
\hline Código & Grado de reacción & $\begin{array}{c}\text { Selecciones } \\
\text { Individuales }\end{array}$ \\
\hline I-127 & 4 & 5 \\
I-126 & 5 & 3 \\
I-107 & 5 & 0 \\
I-102 & 5 & 0 \\
I-123 & 6 & 1 \\
I-135 & 6 & 4 \\
I-163 & 8 & 1 \\
I-167 & 7 & 1 \\
I-251 & 8 & 1
\end{tabular}

\section{Líneas F6}

I-427

$\mathrm{I}-438$

I-455

I-490

I-505

I-614

I-915

I-918

I-916

I-984

I-1028

I- 1040

I-1052

I-1058

I-1064

I-1067

I-1075

I-1092

I-1094

I-1115

I-1132

I-1147

I-1163

I-1174

I-1180

I-1181

I-1189

I-1190

I-1192

\section{Evaluación del Vivero Internacional Caribeño (VICARIBE)}

Este vivero contó con varios tipos de materiales y número de entrada por cada vivero en particular, en el Vivero Internacional Caribeño se evaluaron 73 líneas y donde dos de estas líneas, la PR-9752-74 y PR-9752122 mostraron reacción de resistencia intermedia a la mustia hilachosa con un grado de 4,0 para cada una de las líneas antes mencionadas. Este valor no superó el grado de reacción mostrado por el testigo tolerante 
(Talamanca) que fue de 3,5, pero si superaron ampliamente el testigo susceptible (Barriles) el que mostró un grado de reacción de 6,3, lo que indica el grado de susceptibilidad que tiene este genotipo. Las líneas PR9752-55 y PR - 9752-74 mostraron reacción de 4,7 promedio que superó el valor obtenido por la media del experimento y el obtenido por el testigo susceptible (Cuadro 6). Cabe destacar que estos genotipos poseen fuentes de resistencia al mosaico dorado y a las altas temperaturas que son características importantes en las zonas de producción de frijol en el Caribe.

También fueron evaluadas 78 líneas de frijol rojo moteado. El análisis estadístico practicado a este ensayo mostró diferencias significativas entre los tratamientos $(\mathrm{P} \leq 0,05)$, la línea PR-9745-221 mostró la mejor reacción al ataque del patógeno, con un grado de reacción de 3,3 la que estuvo muy cerca del testigo tolerante (Talamanca) que mostró 3,2 grados de severidad de la mustia hilachosa, las dos líneas, antes mencionadas, están según la escala de evaluación del CIAT como materiales de resistencia intermedia. Un grupo de cinco líneas mostraron buena reacción a la enfermedad (Cuadro 7), estas fueron PR-9745-230 con 3,7, PR,9745-13, PR-9745-226, PR-9750-87 y PR-9750-92 con un grado de reacción de 4,0, indicando lo anterior que estos genotipos tienen resistencia intermedia. Cabe destacar que se según el pedigrí de estas líneas, ya puede tener incorporada la fuente de resistencia a las altas temperaturas y mosaico dorado, por lo que se esperaría que su comportamiento sea bueno en condiciones donde se presentan estas limitantes. Se observa un rápido avance en las líneas caribeñas, debido a que el esfuerzo que se hace en los países de la región se está incorporado al proceso de mejoramiento.

Cuadro 5. Reacción a la Mustia Hilachosa de las líneas sobresalientes evaluadas en el VIDAC- rojo y VIDAC - Negro. Caisán. Panamá.

\begin{tabular}{|c|c|c|c|}
\hline \multicolumn{2}{|c|}{ VIDAC ROJO } & \multicolumn{2}{|c|}{ VIDAC NEGRO } \\
\hline Líneas & Reacción & Líneas $\quad \mathbf{R}$ & Reacción \\
\hline SRC -1-1-18 & 5 & DOR -447 & 4 \\
\hline SRC -1-4-4 & 6 & DOR - 620 & 6 \\
\hline Talamanca (T.T.) & 7 & DOR - 617 & 6 \\
\hline \multirow[t]{8}{*}{ Barriles (T.S.) } & 9 & ICTA JU 95-56 & 6 \\
\hline & & ICTA JU 95-97 & 6 \\
\hline & & ICTA JU 95-91 & 6 \\
\hline & & ICTA JU 95-72 & 6 \\
\hline & & ICTA JU -95-47 & 6 \\
\hline & & DOR 647 & 6 \\
\hline & & TALAMANCA (T.T.) & 7 \\
\hline & & BARRILES (T.S.) & 9 \\
\hline
\end{tabular}

Cuadro 6. Evaluación del vivero de frijol VICARIBE por su resistencia a la mustia hilachosa. Caisán. Panamá. 1997.

\begin{tabular}{llc}
\hline Línea & \multicolumn{1}{c}{ Pedigri } & $\begin{array}{c}\text { Lectura } \\
\text { Mustia }\end{array}$ \\
\hline PR -9752-74 & I-JR// DOR 482/9231-94 & 4,0 \\
PR-9752-122 & DOR 303/T969-2//DOR482/9231-94 & 4,0 \\
PR 9752-55 & DOR 303/T968//DOR482/Belmeb & 4,7 \\
PR 9752-74 & IJR// DOR 482/9231-94 & 4,7 \\
PROMEDIO & & 6,2 \\
TALAMANCA (T.T.) & 3,5 \\
BARRILES (T.S.) & & 6,3 \\
L.S.D. (0.05) & & 1,6 \\
C.V. (\%) & & 15,8 \\
\hline
\end{tabular}

Cuadro 7. Evaluación de líneas de frijol Rojo Moteados Prometedoras por su resistencia a la mustia hilachosa. Caisán. Panamá. 1997.

\begin{tabular}{llr}
\hline Líneas & \multicolumn{1}{c}{ Pedigri } & $\begin{array}{r}\text { Lectura } \\
\text { Mustia }\end{array}$ \\
\hline PR 9745-221 & IJR/9180-25 C//DOR 482/9231-94 & 3.3 \\
PR 9745-230 & IJR/9180-25C//DOR 482/9231-94 & 3.7 \\
PR 9745-13 & M Kelly/ Montealm// DOR 482/9231-94 & 4.0 \\
PR 9745-226 & IJR/9180-25C//DOR 482/9231-94 & 4.0 \\
PR 9750-87 & DOR 482/9231-94//9180-25C & 4.0 \\
PR 9750-92 & DOR 482/9231-94//9180-25C & 4.0 \\
PROMEDIO & & 5.9 \\
TALAMANCA & & \\
(T.T.) & & 3.2 \\
BARRILES (T.S.) & & 6.6 \\
L.S.D.(0-05) & & 1.7 \\
C.V. & & 17.9 \\
\hline
\end{tabular}

Se evaluaron 22 líneas de frijol rojas arriñonadas, pero no hubo diferencias significativas entre los tratamientos, estas líneas mostraron el menor grado de avance. Por lo general las líneas rojas y rosadas, son las que han mostrado la mayor susceptibilidad a este patógeno. El testigo tolerante en el experimento mostró un 3,3 grados de severidad de la enfermedad en tanto que en la variedad Barriles, Testigo susceptible, mostró 6,8 grados de severidad. Las líneas PR-9753-24 y PR9753-124 mostraron 4,3 y 4,7 grados de severidad respectivamente (Cuadro 8 ).

\section{Evaluación del Ensayo Caribeño (ECARIBE)}

Se evaluaron 14 líneas y variedades de frijol, realizando dos lecturas a la presencia de la mustia hilachosa, la primera efectuada a los 46 días de sembrado el frijol y la segunda a los 70 días de sembrado el frijol. Igualmente se registró el peso de campo y el cual fue transformado a rendimientos por hectárea. 
Cuadro 8. Evaluación de líneas de frijol Rojas Arriñonadas por su resistencia a la mustira hilachosa. Caisán. Panamá. 1997.

\begin{tabular}{llr}
\hline Línea & \multicolumn{1}{c}{ Pedigri } & $\begin{array}{c}\text { Lectura } \\
\text { Mustia }\end{array}$ \\
\hline PR 9753-24 & MISS KELLY/MONT CALM// & \\
& DOR 482/19231-94 & 4,3 \\
PR 9753-124 & IJR/9180-294//DOR 482/19251-94 & 4,7 \\
PROMEDIO & & 6,1 \\
TALAMANCA & & \\
(T.T.) & & 3,3 \\
BARRILES (T.S.) & & 6,8 \\
L.S.D.(0.05) & & N.S. \\
CV. (\%) & & 23,2 \\
\hline
\end{tabular}

Para la primera y segunda evaluación de la mustia hilachosa se encontraron diferencias altamente significativas entre los tratamientos ( $\mathrm{P} \leq 0.001)$, así como también para los rendimientos encontrados. Para la primera evaluación de la mustia hilachosa el mejor tratamiento fue la línea MUS-PM-31F5 de origen Dominicano, con 3,0 grados de severidad a la mustia hilachosa, el testigo tolerante, la variedad Talamanca, ocupó un segundo lugar en la evaluación con 3,3 grados de severidad. La variedad Barriles fue la última variedad en esta evaluación, con 7,0 grados de severidad (Cuadro 9). Para la segunda evaluación la línea MUS-PM31F5 y Talamanca ocuparon la primera posición con reacciones de 6,0 para ambos cultivares, seguidos de la línea del programa nacional de frijol de Panamá I-68 con 6,67 grados de severidad de la mustia hilachosa. En el rendimiento la mejor línea fue la variedad I-68 con $3,737.6 \mathrm{~kg} / \mathrm{ha}$ y la línea MUS - PM - 31 F5 con $3,268.8 \mathrm{~kg} / \mathrm{ha}$ (Cuadro 10). La última posición fue ocupada por la variedad Barriles con 124,7 kg/ha Se observa también que el testigo tolerante solamente rindió
Cuadro 10. Rendimiento obtenido por las líneas y su Significancia en el ECARIBE. Caisán. Panamá. 1997.

\begin{tabular}{lc}
\hline Línea & Media y significancia $^{\mathbf{1}}$ \\
\hline I-68 & $3737,6 \mathrm{a}$ \\
MUS-PM - 31F5 & $3268,8 \mathrm{~b}$ \\
PINTO - 6 & $3023,6 \mathrm{bc}$ \\
$9607-29$ & $2755,2 \mathrm{bcd}$ \\
PINTO -1 & $2202,5 \mathrm{cdef}$ \\
9457-43 & $2056,9 \mathrm{defg}$ \\
TALAMANCA (T.T.) & $1844,1 \mathrm{efgh}$ \\
I-62 & $1722,5 \mathrm{fgh}$ \\
IDIAP-R2 (T.L.) & $1233,7 \mathrm{ghi}$ \\
9443-1 & $1101,4 \mathrm{hi}$ \\
9615-10 & $683,4 \mathrm{ij}$ \\
BARRILES & $124,7 \mathrm{j}$ \\
\hline
\end{tabular}

$1 \mathrm{P} \leq 0,001$.

$1,844 \mathrm{~kg} / \mathrm{ha}$ y este tratamiento normalmente produce entre 2,5 y 3,0 t/ha. Para la producción de grano grande en Panamá la línea I-68 continúa con un grado de resistencia alto ante la mustia hilachosa y alta producción. Sin embargo ahora que se abre la ventana de exportación de grano, la línea MUS-PM-31F5, representa una buena alternativa de producción para Panamá.

\section{LITERATURA CITADA}

BEEBE, S. 1986. Mejoramiento genético para resistencia a la mustia hilachosa In: Memorias del Segundo Taller de Mustia hilachosa. San José Costa Rica. 124 p.

FLENTJE, N.T. ; DODMAN, R.L.; KERR. A. 1963. The mechanisn of host penetration by Thanatephorus сисиmeris. Aust. J. Biol. Sci. 16: 784-799.

Cuadro 9. Separación de medias para la primera y segunda lectura de la severidad de la mustia hilachosa en el ECARIBE. Caisán. Panamá. 1997.

\begin{tabular}{lclc}
\hline Tratamiento & Medias para mustia & \multicolumn{1}{c}{ Tratamiento } & Medias para mustia \\
\hline MUS - PM -31F5 & $3,00 \mathrm{a}$ & Mus -PM- 31 F5 & $6,0 \mathrm{a}$ \\
TALAMANCA (T.T.) & $3,33 \mathrm{ab}$ & TALAMANCA (T.T.) & $6,00 \mathrm{a}$ \\
$9457-43$ & $4,33 \mathrm{abc}$ & I.68 & $6,67 \mathrm{ab}$ \\
PINTO-6 & $4,50 \mathrm{abc}$ & PINTO 6 & $7,00 \mathrm{abc}$ \\
I-68 & $4,66 \mathrm{abc}$ & $9457-43$ & $7,33 \mathrm{abcd}$ \\
I-62 & $5,00 \mathrm{bcd}$ & $9607-29$ & $7,33 \mathrm{abcd}$ \\
$9607-29$ & $5,00 \mathrm{bcd}$ & PINTO 1 & $7,50 \mathrm{abcd}$ \\
IDIAP-R2 (T.L.) & $5,66 \mathrm{cde}$ & IDIAP-R2 (T.L) & $7,66 \mathrm{bcd}$ \\
9443-1 & $5,66 \mathrm{cde}$ & I-62 & $7,66 \mathrm{bcd}$ \\
PINTO 1 & $6,00 \mathrm{cde}$ & $9443-1$ & $8,33 \mathrm{~cd}$ \\
$9615-10$ & $6,66 \mathrm{de}$ & $9615-10$ & $8,33 \mathrm{~cd}$ \\
BARRILES (T.S.) & $7,00 \mathrm{e}$ & BARRILES & $8,66 \mathrm{~d}$ \\
\hline
\end{tabular}

$1 \mathrm{P} \leq 0,001$. 
GALINDO, J.J. 1982. Epidemiology and control of web blight of bean in CostaRica. Tesis (Ph.D), Cornell University, Ithaca, N. Y. E. U: 141 p.

GALVEZ, G. E. ; MORA, B.;PASTOR,M. 1994. La mustia hilachosa In: Problemas de pudrición del frijol en los trópicos. CIAT. Cali. Colombia. p. 227.

PASTOR-C., M. 1985. Enfermedades del frijol causadas por hongos. In: Frijol: investigación y producción. CIAT p. 186.

PROFRIJOL. 1997. Informe técnico por resultados. POA 1996-97. Guatemala. 57 p.
RODRÍGUEZ, E.; GONZÁLEZ,F. 1998. Mejoramiento genético del frijol andino para la resistencia a la mustia hilachosa. In: Memorias del taller de mejoramiento del Frijol Negro. Veracruz, México. PROFRIJOL.

RODRÍGUEZ, E.; DE GRACIA, R.; GONZÁLEZ, F. 1995. Poroto (Phaseolus vulgaris). Guía técnica para su cultivo. IDIAP. Panamá. 27 p.

RODRÍGUEZ, E. ; LORENZO.E.; DE GRACIA, R.; GONZÁLEZ, G.; GONZÁLEZ, F. 1997. Manual técnico. Manejo integrado del cultivo de frijol común o poroto (Phaseolus vulgaris L) en el sistema de mínima labranza. IDIAP. Panamá. 75 p. 